\title{
Automatic Gas Valve Control System using Arduino Hardware
}

\author{
T.K. Sethuramalingam and M. Karthighairasan
}

\begin{abstract}
In this paper, the automatic gas valve control system is designed using arduino hardware. Arduino is an open hardware platform that provides a rapid prototype development. A servomotor is fitted to a gas valve. Four control positions are carried out by the module, two main positions are used to open or close the valve. The valve opens at $90^{\circ}$ and closes to $0^{\circ}$. This library allows an Arduino board to control RC (hobby) servo motors. Servos have integrated gears and a shaft that can be precisely controlled.
\end{abstract}

Keywords--- Arduino, Servomotor, Integrated Gears, Shafts

\section{INTRODUCTION}

A RDUINO is an open hardware platform which is a portion of the automatic control for laboratory sterilization progression. Sterilization is a familiar practice in biological laboratories and it is defined as a development of microbial grown control, concerning the overall annihilation of any microorganism on all objects. Diverse techniques exploit heat as a method to demolish microorganisms. These are referred as thermal techniques. Two main techniques are moist heating and dry heating. Moist heating makes use of water steam. This module is exploited for controlling the flame for producing heat.

\section{ARDUINO MICROCONTROLLER}

The Arduino Uno is a microcontroller board depending on the ATmega328. It includes 14 digital input/output pins (out of which 6 can be exploited as PWM outputs), 6 analog inputs, a $16 \mathrm{MHz}$ crystal oscillator, a USB connection, a power jack, an ICSP header and the last one is a reset button. It includes the most necessary things which are essential to support the microcontroller; basically attach it to a computer with a USB cable or power it with an AC-to-DC adapter or battery to get started.

Arduino is an open - source single board microcontroller, intended to formulate a process of exploiting electronics in multidisciplinary projects more accessible.

The hardware comprises of a simple open hardware devise for the Arduino board with an Atmel processor and on-board I/O support. The software comprises of a standard programming language and also the boot loader that runs on the board.

T.K. Sethuramalingam, M.Tech (Embedded Systems) Final year, Department of Electronics \& Communication Engineering, PRIST University, Trichy Campus, Tamilnadu, India. E-mail: tksethuramalingam@gmail.com

M. Karthighairasan, Assistant Professor and Head, Department of Electronics \& Communication Engineering, PRIST University, Trichy Campus, Tamilnadu, India. E-mail: mkrasan@gmail.com
Arduino hardware is programmed with the help of a wring-dependent language (syntax + libraries), comparable to $\mathrm{C}++$ with some simplifications and adaptations, and a processing-dependent IDE.

The Uno is different from all previous boards in that it does not employ the FTDI USB-to-serial driver chip. As an alternative, it features the Atmega16U2 (Atmega8U2 up to version R2) programmed as a USB-to-serial converter. "Uno" represents one in Italian and is named to mark the forthcoming release of Arduino 1.0. The Uno and version 1.0 will be the reference versions of Arduino, moving forward. The Uno is the most recent in a series of USB Arduino boards, and the reference model for the Arduino platform.

\section{ARDUINO HARDWARE}

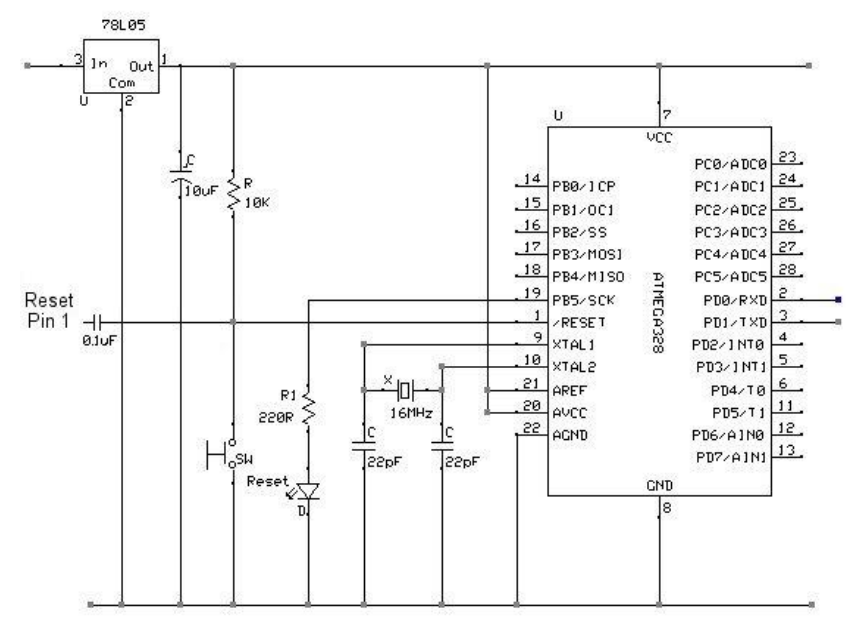

Figure 1: Arduino Uno schematic diagram

An Arduino board comprises of an 8-bit Atmel AVR microcontroller with constituents entirely to assist programming and integration into other circuits. A significant characteristic of the Arduino is the standard technique that connectors are uncovered, permitting the CPU board to be connected to a range of interchangeable add-on modules (known as shields). Official Arduino have employed the mega AVR series of chips, particularly the ATmega8, ATmega168, ATmega328 and ATmega1280. A handful of other processor has been utilized by Arduino compatibles. 


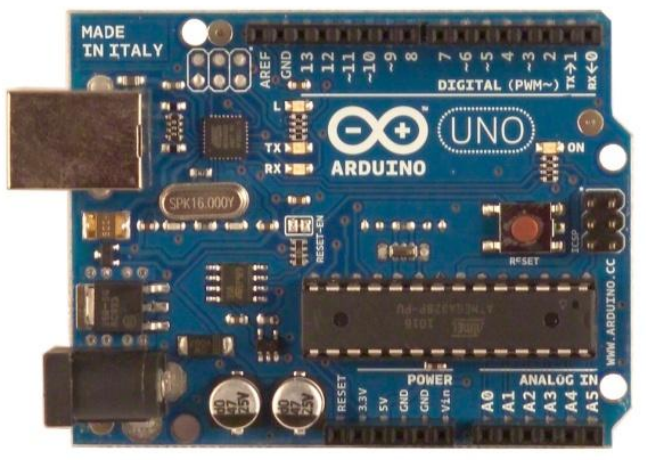

Figure 2: Arduino Uno Hardware

The majority of the boards incorporate a 5 volt linear regulator and a $16 \mathrm{MHZ}$ crystal oscillator (or ceramic resonator in a few variants), even though certain designs such as the Lily pad run at $8 \mathrm{MHZ}$ and dispense with the onboard voltage regulator because of particular form-factor limitations. In addition, an Arduino microcontroller is preprogrammed with a boot loader that simplifies uploading of programs to the on-chip flash memory, evaluated with other devices that characteristically require an external chip programmer.

At an intangible level, when exploiting the Arduino software stack, the entire boards are programmed over an RS-232 serial connection, however the method this is implemented differs by hardware version. Serial Arduino boards include an uncomplicated inverter circuit to exchange between RS-232 level and TTL level signals. Current Arduino boards are programmed by means of USB, implemented using USB-to-serial adapter chips, for instance, the FTDI FT232. A few variants, such as the Arduino mini and the unofficial board uno, employ a detectable USB-to-serial adapter board or cable, Bluetooth or other techniques. When exploited with conventional microcontroller tools in place of the Arduino IDE, standard AVR ISP programming is employed. The Arduino board exposes most of the microcontroller's I/O pins for use by other circuits.

\section{FeAtures of ATMEGA328}

The controller comprises of $8 \mathrm{~K}$ bytes of In-System Programmable Flash with Read-While-Write capabilities, 512 bytes of EEPROM, 1K byte of SRAM, 23 universal purpose I/O lines, 32 common purpose working registers, 3 flexible Timer/Counters with balance modes, internal and external interrupts, a serial programmable USART, a byte oriented Two-wire Serial Interface, a 6-channel ADC (eight channels in TQFP and QFN/MLF packages) with 10-bit accuracy, a programmable Watchdog Timer with Internal Oscillator, an SPI serial port and 5 software adjustable power saving modes. The idle mode discontinue the CPU while permitting the SRAM, Timer/Counters, SPI port and suspend system to continue functioning.

The power down mode accumulates the register contents but freezes the Oscillator, immobilizing all other chip functions until the next Interrupt or Hardware Reset. In Power-save mode, the asynchronous timer continues to run, permitting the user to continue a timer base at the same time the rest of the device is sleeping. The ADC Noise Reduction mode discontinues the $\mathrm{CPU}$ and the entire I/O modules excluding asynchronous timer and $\mathrm{ADC}$, to reduce switching noise throughout ADC conversions. In Standby mode, the crystal/resonator Oscillator is running at the same time the remaining device is sleeping. This facilitates very fast start-up integrated with low-power consumption.

The device is constructed with the help of Atmel's high density non-volatile memory technology. The Flash Program memory can be reprogrammed In-System through an SPI serial interface, by a traditional non-volatile memory programmer, or by an On-chip boot program running on the AVR core. The boot program can employ any interface to download the application program in the Application Flash memory. Software in the Boot Flash Section will prolong to run at the same time the Application Flash Section is updated, providing true Read-While-Write operation. By integrating an 8-bit RISC CPU with In-System SelfProgrammable Flash on a monolithic chip, the Atmel ATMEGA328 is a dominant microcontroller that offers an extremely-flexible and cost-effective solution to several embedded control applications.

The ATMEGA328 AVR is supported with a complete suite of program and system development tools, together with C compilers, macro assemblers, program debugger/simulators, In-Circuit Emulators, and evaluation kits.

This set of connections has other implications. When the Uno is linked to either a computer running Mac OS X or LINUX, it resets each time a connection is made to it from software (via USB). For the following half-second or so, the boot loader is running on the Uno. At the same time it is programmed to disregard distorted data (i.e. everything besides an upload of new code), it will break up the initial few bytes of data receives one-time configuration or additional data when it starts initially, make sure that the software with which it communicate waits a second after opening the connection and before sending this data.

The Uno includes a trace that can be cut to immobilize the auto-reset. The pads on either side of the trace can be soldered jointly to re-enable it. It's labelled "RESET-EN". It is also possible to disable the auto-reset by connecting a 110 ohm resistor from $5 \mathrm{~V}$ to the reset line.

\section{Gas Valve Control Module}

A servomotor fixed to a gas valve. Four control positions are carried out by the module, two major positions are exploited to open or close the valve (Open at $90^{\circ}$ and close to $0^{\circ}$ ).

This library allows an Arduino board to manage RC (hobby) servo motors. Servos have incorporated gears and a shaft that can be accurately controlled. Standard servos permit the shaft to be positioned at different angles, typically between 0 and 180 degrees. Uninterrupted rotation servos permit the rotation of the shaft to be set to different speeds.

The Servo library supports up to 12 motors on most Arduino boards and 48 on the Arduino Mega. On boards excluding the Mega, make use of the library disables analog Write() (PWM) functionality on pins 9 and 10, whether or not there is a Servo on those pins. On the Mega, up to 12 servos can be exploited without interfering with PWM 
functionality; exploitation of 12 to 23 motors will immobilize PWM on pins 11 and 12.

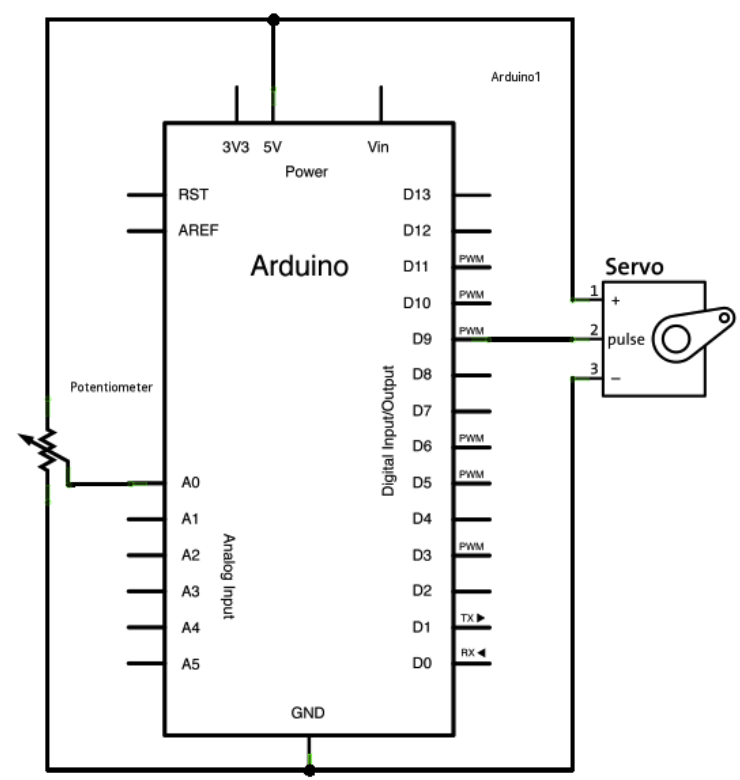

Figure 3: Servo motor interfacing using arduino

Servo motors consists of three wires: power, ground and signal. In general, the power wire is red in colour and supposed to be connected to the $5 \mathrm{~V}$ pin on the Arduino board. The ground wire is characteristically black or brown and must be connected to a ground pin on the Arduino board. The signal pin is normally yellow or orange and should be connected to pin 9 on the Arduino board.

The potentiometer is supposed to be wired in order that its two outer pins are connected to power $(+5 \mathrm{~V})$ and ground, and its center pin is connected to analog input 0 on the Arduino.

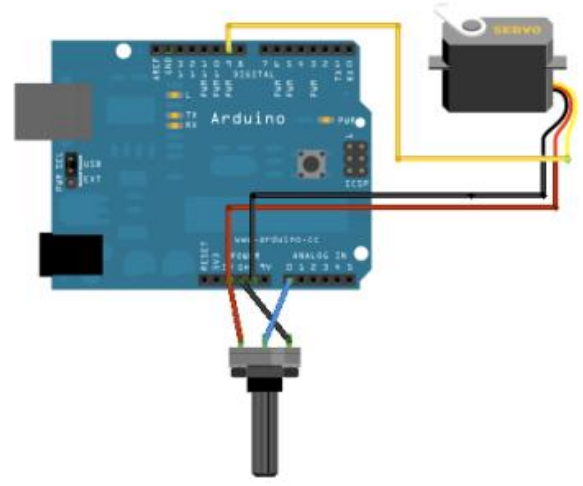

Figure 4: Servo Motor Connection Diagram

\section{ARDUINO DEVELOPMENT ENVIRONMENT}

The Arduino development environment comprises of a text editor for writing code, a message area, a text console, a toolbar with buttons for frequent functions and a sequence of menus. It attaches to the Arduino hardware to upload programs and communicate with them.

\section{A. Language Editor}

The Arduino 1.0.1 software atmosphere has been interpreted into $30+$ different languages. In normal case, the IDE loads in the language chosen by our operating system. The language can be manually adjusted by starting the
Arduino software and selecting the Preferences window. Adjacent to the editor language there is a dropdown menu of currently supported languages. The desired language can be chosen from the menu and restart the software to make use of the preferred language. If the desired language is not supported, the IDE will default to English.

The Arduino can return to its normal setting depending on the operating system by selecting System Default from the Editor Language drop-down. This setting will commence only after restarting the Arduino software. In the same way, after changing the operating system's settings, the Arduino software must be restarted to update it to the latest default language.

\section{B. Writing Sketches}

Software is written using Arduino which are called as sketches. These sketches are written in the text editor. Sketches are saved with the file extension as .ino. It has options for cutting/pasting and for searching/replacing text. The message area provides feedback at the time of saving and exporting and also displays errors. The console shows text output by the Arduino environment together with complete error messages and additional information. The bottom right-hand corner of the window shows the existing board and serial port. The toolbar button permits to confirm and upload programs, create, open and save sketches and open the serial monitor.

\section{Sketchbook}

The Arduino environment employs the idea of a sketchbook - a standard place to accumulate the programs (or sketches). The sketches in sketchbook can be initialized from the File > Sketchbook menu or from the Open button on the toolbar. The initial time while running the Arduino software, it will automatically generate a directory for the sketchbook. The location of the sketchbook can be viewed or modified with the assistance of Preferences dialog.

\section{Libraries}

Libraries offer additional functionality for use in sketches, for instance, working with hardware or manipulating data. In order to use a library in sketch, it is necessary to select it from the Sketch > Import Library menu. This will introduce one or more \#include statements at the beginning of the sketch and compile the library with the sketch. Since the libraries are uploaded to the board with the sketch, they enhance the quantity of space it takes up. When a sketch no longer requires a library, just remove its \#include statements from the top of the code.

\section{CONCLUSION}

In this paper, the gas valve open and close position was effectively implemented. Moreover, this module will be implemented in the automatic control for laboratory sterilization process.

\section{REFERENCES}

[1] Arizaga, J.A. de la Calleja, J. ; Hernandez, R. ; Benitez, A. Univ. Politec. de Puebla, Puebla, Mexico, Automatic Control for Laboratory Sterilization Process based on Arduino Hardware, 22nd International Conference on Electrical Communications and Computers (CONIELECOMP), 2012 pg. 130 - 133

[2] Zualkernan, I.A. InfoCoral: Open-Source Hardware for Low-Cost, High-Density Concurrent Simple Response Ubiquitous Systems; 2011 
11th IEEE International Conference on Advanced Learning Technologies (ICALT), Page(s): 638 - 639

[3] Al-Kuwari, A.M.A.H.; Ortega-Sanchez, C.; Sharif, A.; Potdar, V.User friendly smart home infrastructure: BeeHouse; 2011 Proceedings of the 5th IEEE International Conference on Digital Ecosystems and Technologies Conference (DEST). Page(s): 257 - 262

[4] Che-Yuan Tu; Wen-Chieh Kuo; Wei-Hua Teng; Yao-Tsung Wang; Shiau, S.; A Power-Aware Cloud Architecture with Smart Metering. 39th International Conference on Parallel Processing Workshops (ICPPW) 2010 Page(s): 497 - 503

[5] Michael Morgolis - Arduino cookbook - II Edition 2012, O’Reilly Publications

[6] http://arduino.cc/en/Main/ArduinoBoardUno

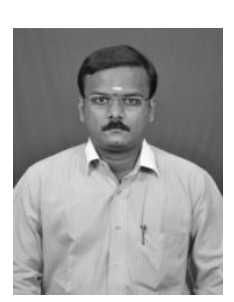

T.K. Sethuramalingam, This author was born in Tamil Nadu, India, in 1981 and received the B.Sc. degree from the Manonmaniam Sundaranar University, India, in 2001. Further he received his M.Sc. and M.Phil. degrees from Bharathidasan University, India, in 2003 and 2005. He is currently pursuing his M.Tech project work under the guidance of Mr. M.Karthighairasan. He visited foreign countries and presented his research publications. He is a Member in IEEE, ISSS, IACSIT, IETE, ISTE.His research interests and publications have been in the areas of Embedded systems, VLSI design and MEMS.

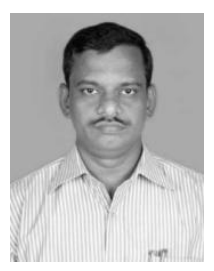

M. Karthighairasan, This author was born in Tamil Nadu, India, in 1975. He received his B.E. (Electronics and Communication) in 2002 from Regional Engineering College, Tiruchirappalli. Further he received his M.Tech. (Advanced Communication Systems) in 2005 from Shanmugha Arts, Science, and Technology \& Research Academy (SASTRA University) Thanjavur, India. Currently he is working as Assistant Professor and Head in Department of Electronics and Communication Engineering at PRIST University, Tiruchirappalli Campus, Tiruchirappalli, India He has more than 7 years of teaching experience. His research interests and publications have been in the areas of Satellite and Wireless Communications. 